\title{
OPEN SOURCE CABLE MODELS FOR EMI SIMULATIONS
}

\author{
S. Greedy ${ }^{1}$, C. Smartt ${ }^{1}$, D. W. P. Thomas ${ }^{1}$. \\ 1. George Green Institute for Electromagnetics Research, Department of Electrical and Electronic Engineering, \\ University of Nottingham, NG7 2RD, steve.greedy@nottingham.ac.uk
}

\begin{abstract}
This paper describes the progress of work towards an Open Source software toolset suitable for developing Spice based multi-conductor cable models. The issues related to creating a transmission line model for implementation in Spice which include the frequency dependent properties of real cables are presented and the viability of spice cable models is demonstrated through application to a three conductor crosstalk model. Development of the techniques to include models of shielded cables and incident field excitation has been demonstrated.
\end{abstract}

\section{INTRODUCTION}

There has been a significant amount of progress in the development of software based simulation tools that focused on advanced models of cables and multiconductor transmission lines (MTL) [1,2]. These models can include the effects of frequency dependent cable properties, imperfect shielding and external field to cable coupling mechanisms. However advanced cable models are either not readily available to the wider EMC community or come at significant cost in terms of both procurement and effort required to learn how to use these tools effectively. These issues are being addressed by the development of sophisticated Spice based multi-conductor transmission line models that implement the state-of-the-art in cable simulation techniques in a form of a toolset that will be made available through an open source license.

From the outset the development has been governed by the requirement for an accurate, robust, numerically efficient model with the following features:

- Time domain and frequency domain simulation capabilities

- Ability to describe frequency dependent cable properties

- Models of shielded cables, with transfer impedance coupling, including multiple levels of shielding

- Twisted pair cable model

- Capability for the inclusion of coupling onto cables from external electromagnetic field sources

- Portability between Ngspice, LTSpice and PSpice

\section{METHODOLOGY}

A MTL is assumed to comprise multiple cables that may or may not feature over-shields and a ground plane. Individual cables within the bundle will have frequency dependent characteristics and each of these individual cables may themselves include shielded conductors as illustrated in Fig. 1.

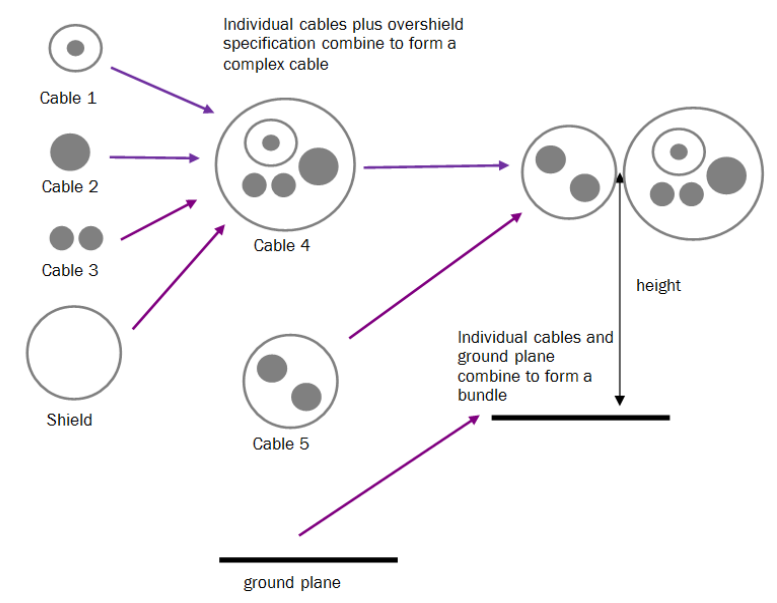

Figure 1. Illustration of a cable bundle built with multiple layers of shielding.

In developing the Spice model the multi-conductor propagation model based on modal decomposition is used [1]. The frequency dependence of mode propagation on the multi-conductor system is accounted for through the method of characteristics with the inclusion of a propagation correction. The propagation within shields is further dealt with by domain decomposition and coupling across imperfect shields and is accounted for via a weak form of the transfer impedance coupling [3]. External incident field excitation is assumed to be in the form of a plane wave.

If we assume that we wish to model a single mode transmission line whose properties are described by frequency dependent inductance and resistance, frequency independent capacitance and zero admittance. The frequency dependence of the inductance and the resistance is assumed to be due to the penetration of the field into the conductors due to their finite electrical conductivity. The total inductance may be separated into 
two components, the first due to the magnetic field outside the conductors which is assumed to be frequency independent and the second due to the magnetic field within the conductors. Similarly the resistance is separated into a d.c. resistance and a frequency dependent resistance. Eq. 1.

$$
\begin{aligned}
& L=L_{0}+L_{\text {int }}(j \omega) \\
& R=R_{d c}+R(j \omega) \\
& C=C_{0}
\end{aligned}
$$

The internal impedance for a cylindrical conductor, assuming a uniform current distribution around the conductor circumference may be calculated as, Eq. 2 .

$$
Z_{\text {int }}=\frac{1}{\sqrt{2} \pi r_{w} \sigma \delta}\left(\frac{\operatorname{ber}(q)+j b e i(q)}{\operatorname{bei}^{\prime}(q)-j \operatorname{ber}^{\prime}(q)}\right)
$$

where

$$
\delta=\frac{1}{\sqrt{\pi f \mu \sigma}} \quad \text { and } \quad q=\sqrt{2} \frac{r_{w}}{\delta}
$$

For a test case of a single cable where $\mathrm{L} 0=1.727 \mu \mathrm{H} / \mathrm{m}$ $\mathrm{C} 0=6.440 \mathrm{pF} / \mathrm{m}, \mathrm{Rdc}=0.558 \Omega / \mathrm{m}$ on each conductor and $\mathrm{Z} 0=518 \Omega$, the calculated internal impedance is shown in Fig. 2.

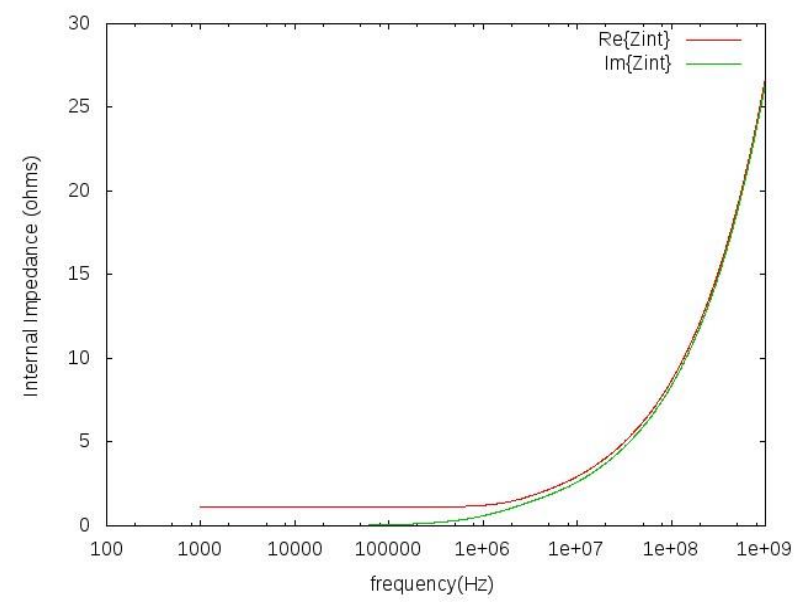

Figure 2. Internal impedance as a function of frequency.

Figure 3 shows the characteristic impedance of the transmission line given by Eq. 4 and the high frequency limit of the impedance given by Eq. 5 .

$$
\begin{gathered}
Z_{c}(j \omega)=\frac{\sqrt{R(j \omega)+j \omega L(j \omega)}}{\sqrt{j \omega C(j \omega)}} \\
Z_{0}=\sqrt{\frac{L_{0}}{C_{0}}}
\end{gathered}
$$

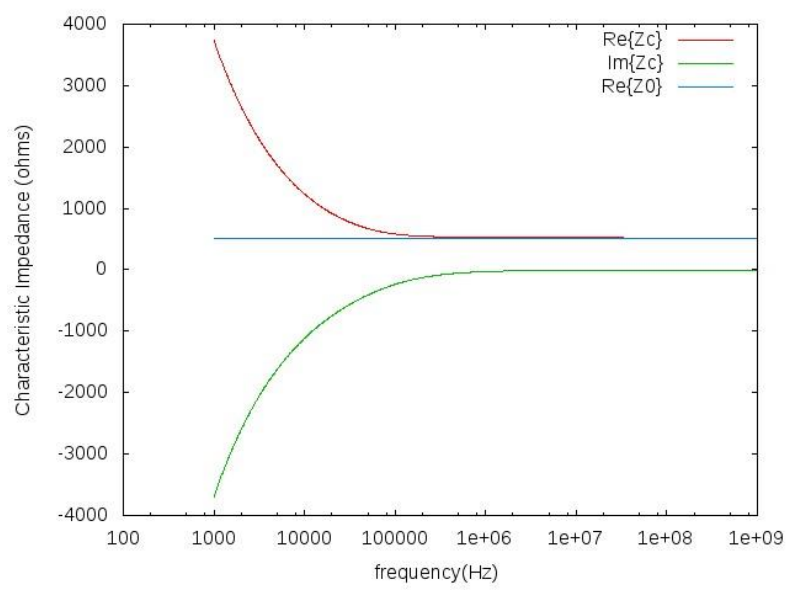

Figure 3. Characteristic impedance as a function of frequency.

Therefore a proposed propagation model which has the correct low frequency behaviour and includes frequency dependent loss is shown in Fig. 4.

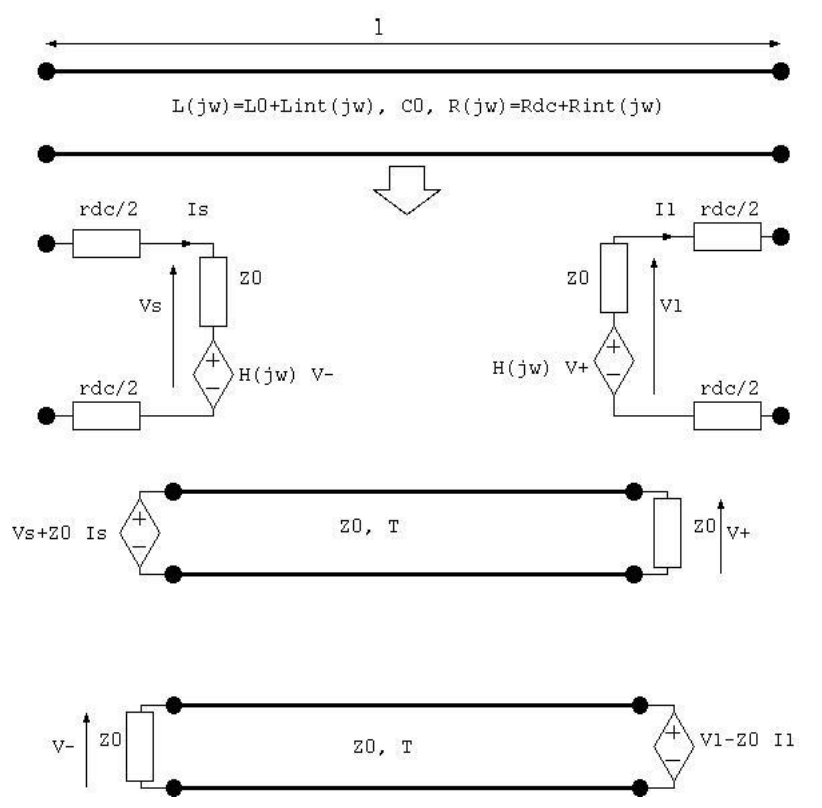

Figure 4. Circuit model of a transmission line with frequency dependent properties suitable for implementation in Spice.

The model has the following features which make it suitable for Spice simulations:

- D.C. resistance is included in the model - this is included as lumped resistances placed on each conductor at either end of the transmission line

- Transmission delay is modelled using a lossless transmission line with the (frequency independent) 'high frequency' characteristic impedance, Z0. The model is based on the 
method of characteristics and two transmission lines are required in the model, one for each propagation direction.

- The transmitted waves are corrected for dispersion and attenuation by a frequency dependent transfer function $\mathrm{H}(\mathrm{j} \omega)$. This transfer function may be implemented in Spice using the lumped s-domain transfer function i.e. $\mathrm{H}(\mathrm{s})$ where $\mathrm{H}(\mathrm{s})$ takes the form of a rational function of $s$.

- If the correction is neglected the model reduces to the lossless model described by Paul [4].

The propagation correction may be included into the model in two parts i.e. $\sqrt{ } \mathrm{H}(\mathrm{j} \omega)$ applied at the source end of the characteristic propagation delay line and $\sqrt{ } \mathrm{H}(\mathrm{j} \omega)$ at the termination. This gives a symmetrical (and reciprocal) system and should improve the accuracy of the transfer impedance and incident field excitation models at the expense of additional complexity in the Spice circuit model.

Validation of the method for a two conductor transmission line, Fig. 5, with frequency dependent skin effect model shows that the model can reproduce the behaviour of the original transmission line with a high degree of accuracy over the frequency band of interest in this study i.e. d.c. to $1 \mathrm{GHz}$.

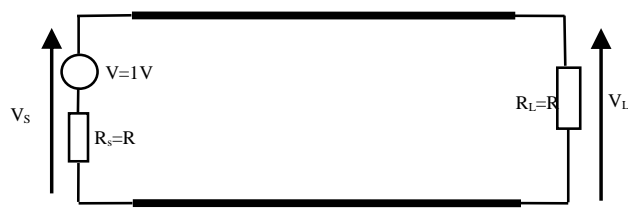

Figure 5. Two conductor transmission line test configuration.

Fig. 6 to Fig. 8 below show load end termination voltage results for the low impedance source and load resistance test case for a number of different degrees of approximation in the model. These are:

- Lossless model: The series resistance and internal inductance are assumed to be zero. Fig. 4 . shows that the lossless model is inaccurate at low frequency and at high frequency.

- Model with d.c. resistance added: The d.c. resistance of the conductors is added at the terminations. No frequency dependent resistance or inductance is included. Figure 2.8 and Fig. 5 shows that including the d.c. resistance corrects the low frequency results however the high frequency results remain inaccurate.

- Frequency dependent method of characteristics solution. The model includes the d.c. resistance and the frequency dependent propagation correction. Fig. 6 shows that the combination of the d.c. resistance and the propagation correction in the model gives results which are accurate across the whole frequency range of analysis.

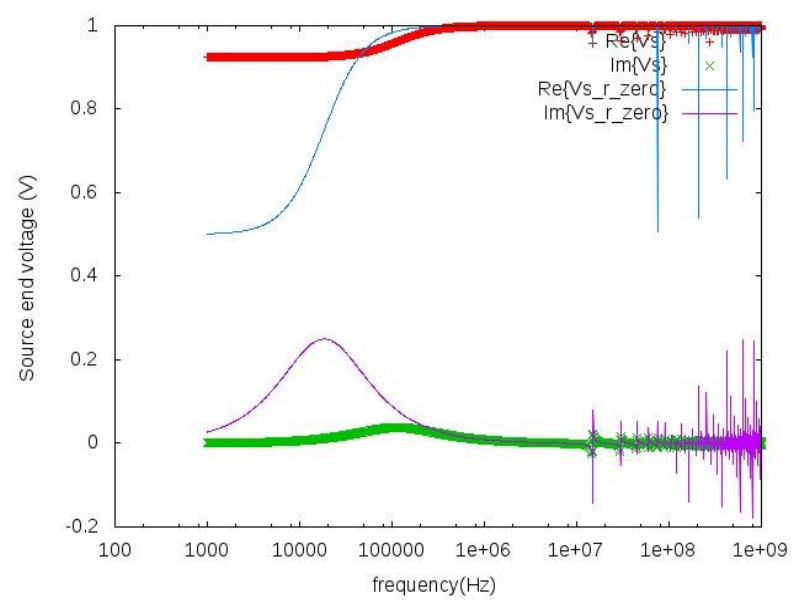

Figure 6. Comparison of exact and lossless model solution: source end voltage.

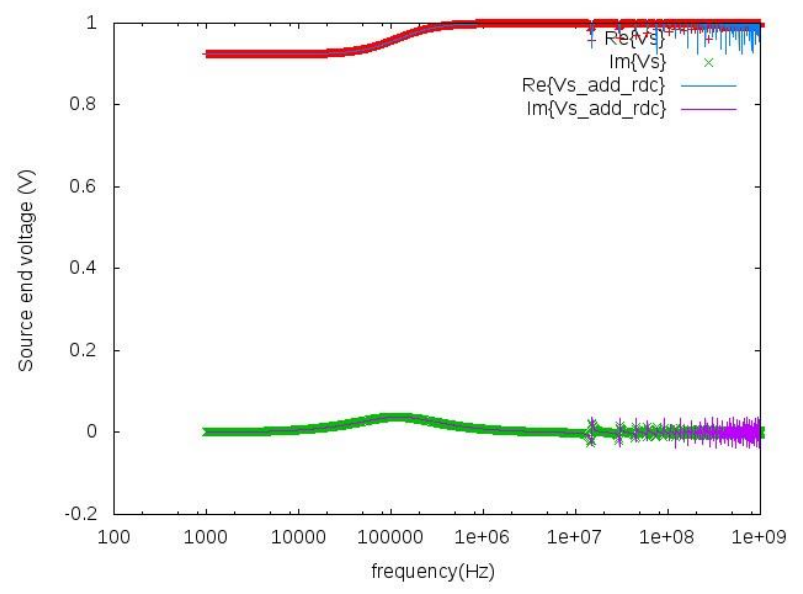

Figure 7. Comparison of exact and model with d.c. resistance added: source end voltage.

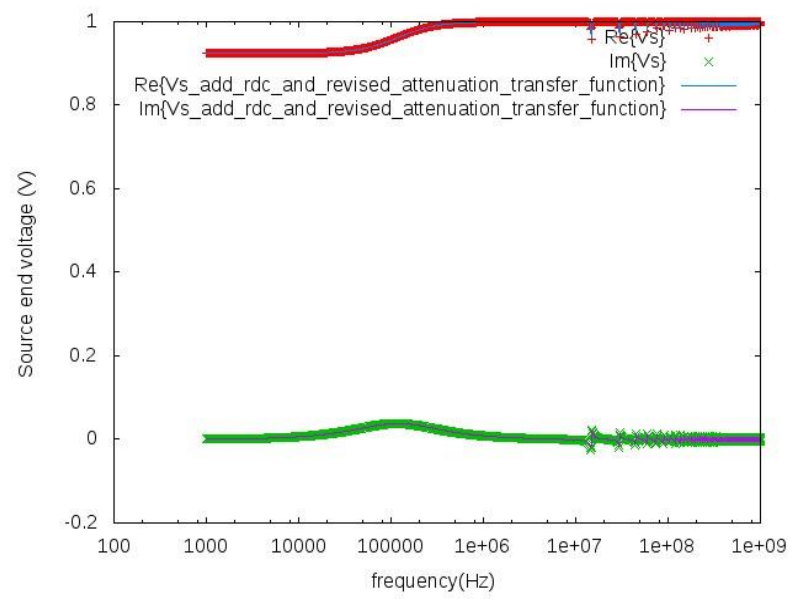

Figure 8. Exact and frequency dependent method of characteristics solution. 


\section{RESULTS}

An implementation of the proposed model in Ngspice is demonstrated here so as to give confidence that it is capable of modelling frequency dependent propagation on multi-conductor transmission lines. The frequency dependence is included in the model as a s-domain impedance function, $Z_{\text {int }}$ Eq. 6, which appears in series with the inductive impedance of the transmission line.

$$
Z_{\text {int }}=\frac{a_{0}+a_{1}\left(\frac{j \omega}{\omega_{0}}\right)+a_{2}\left(\frac{j \omega}{\omega_{0}}\right)^{2}+a_{3}\left(\frac{j \omega}{\omega_{0}}\right)^{3}+a_{4}\left(\frac{j \omega}{\omega_{0}}\right)^{4}}{b_{0}+b_{1}\left(\frac{j \omega}{\omega_{0}}\right)+b_{2}\left(\frac{j \omega}{\omega_{0}}\right)^{2}+b_{3}\left(\frac{j \omega}{\omega_{0}}\right)^{3}+b_{4}\left(\frac{j \omega}{\omega_{0}}\right)^{4}}
$$

To test and validate the approach a three conductor configuration, Fig. 9, with frequency dependent loss was used. The model includes a 'modal transformer' in order to perform a modal decomposition. The complete specification for the cables, including the filter parameters used in Eq. 6, is given in Tab. 1.

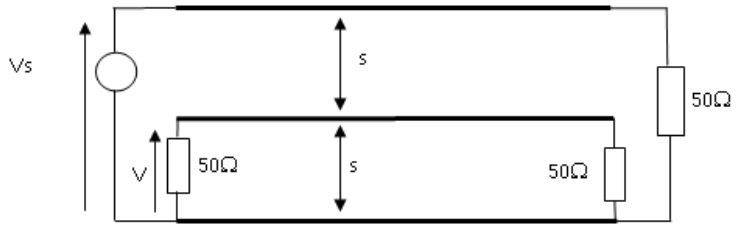

Figure 9. Three conductor crosstalk configuration.

\begin{tabular}{|c|c|}
\hline \multicolumn{2}{|c|}{3 Cylindrical conductors with dielectric coating: } \\
\hline Conductor radius & $0.1905 \mathrm{~mm}$ \\
\hline Dielectric radius & $0.4445 \mathrm{~mm}$ \\
\hline $\begin{array}{lll}\begin{array}{l}\text { relative } \\
\text { dielectric }\end{array} & \text { permittivity } & \text { of } \\
\end{array}$ & 3.5 \\
\hline conductor separation, $\mathrm{s}$ & $1.27 \mathrm{~mm}$ \\
\hline transmission line length & $2.0 \mathrm{~m}$ \\
\hline \multicolumn{2}{|c|}{ Series impedance filter parameters: } \\
\hline$\omega_{0}$ & $2 \pi \times 10^{9}$ \\
\hline $\mathrm{a}_{0}$ & 0.1585182 \\
\hline$a_{1}$ & 37.97350 \\
\hline $\mathrm{a}_{2}$ & 731.8634 \\
\hline $\mathrm{a}_{3}$ & 1974.191 \\
\hline $\mathrm{a}_{4}$ & 782.3851 \\
\hline $\mathrm{b}_{0}$ & 1.0 \\
\hline $\mathrm{b}_{1}$ & 67.24337 \\
\hline$b_{2}$ & 479.6192 \\
\hline$b_{3}$ & 518.7956 \\
\hline $\mathrm{b}_{4}$ & 60.77901 \\
\hline
\end{tabular}

Table 1. Cable specification for 3 conductor test case.

The near end crosstalk is calculated for this configuration the results of the frequency dependent model described here are plotted against the analytic solution. The results show the correct behaviour at low frequency, <100MHz, Fig. 10.

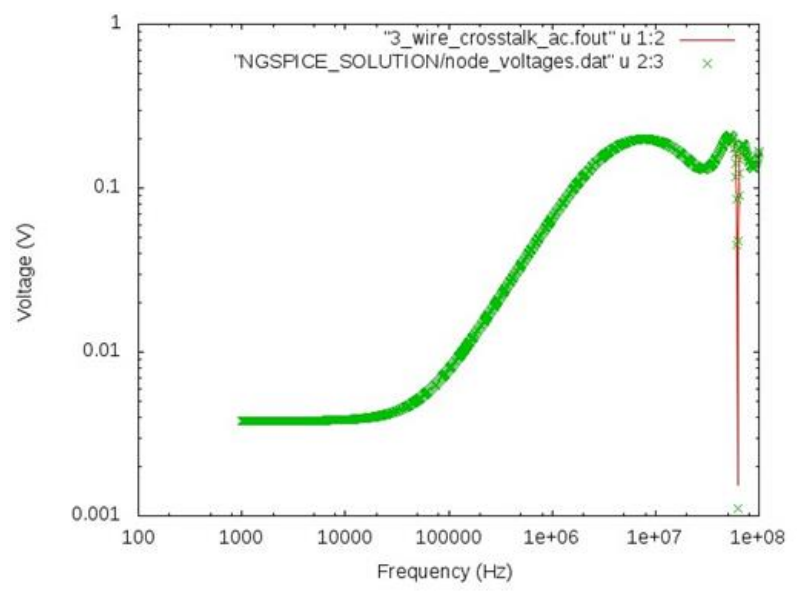

Figure 10. Near end crosstalk calculation: Analytic solution and Spice model result.

Fig. 11(a) and Fig. 11(b) show the near end crosstalk with a linear frequency scale up to $1 \mathrm{GHz}$ without and with the propagation correction (s-domain transfer function) included in the Spice model where is demonstrated that the inclusion of correction improves the agreement between the Spice model and the measurement.

(a)

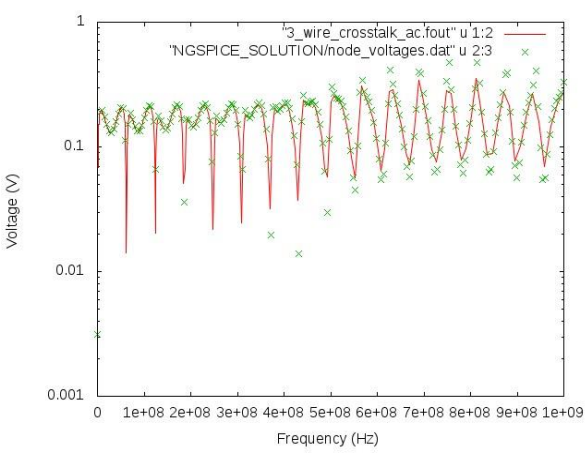

(b)

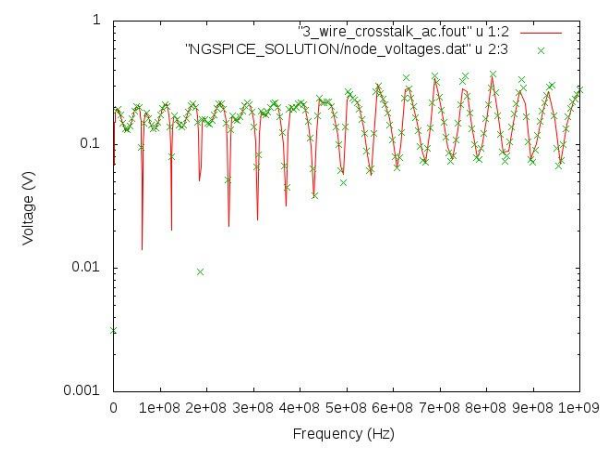

Figure 11. Comparison of Analytic solution and Spice model (a) without and (b) with frequency dependent parameter correction. 
Next a test configuration consisting of three parallel cylindrical conductors, Fig. 12, illuminated by an external $E$ field which is polarised along the transmission line and the $\mathrm{k}$ vector is in the plane of the transmission line, perpendicular to the line. The incident field excitation model is based on that published in [4]. The cable is parameters for this configuration are given in Table 2. The results are presented in Fig. 13.

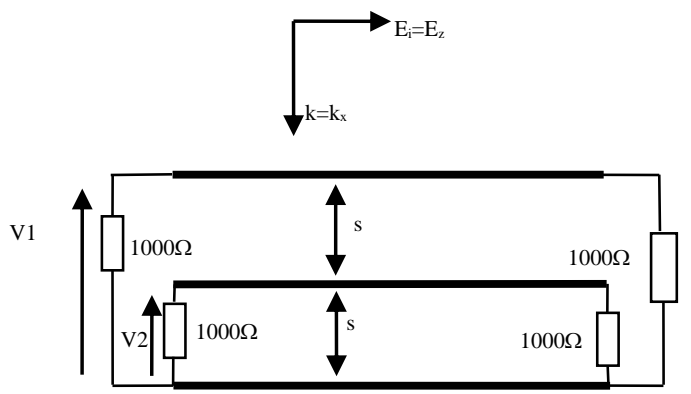

Figure 12. Three wire incident field configuration.

\section{Cylindrical conductors with no dielectric coating}

\begin{tabular}{|l|l|}
\hline Conductor radius & $1 \mathrm{~mm}$ \\
\hline conductor separation, $\mathrm{s}$ & $1 \mathrm{~cm}$ \\
\hline transmission line length & $1.0 \mathrm{~m}$ \\
\hline \multicolumn{2}{|l}{ No frequency dependent loss present } \\
\hline
\end{tabular}

Table 2. Cable specification for 3 conductor test case in presence of external field.

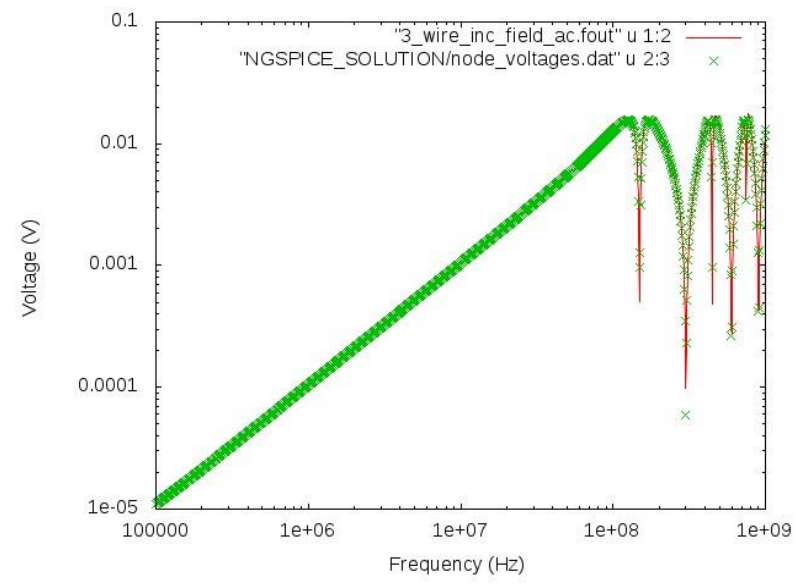

Figure 13. Coupling from incident field to multiconductor transmission line.

This problem was then run in a transient simulation mode and the results are shown in Fig. 14. Note that
Ngspice fails to run to completion in a reasonable time if the transient time required is much greater than $10 \mu \mathrm{s}$ for this case.

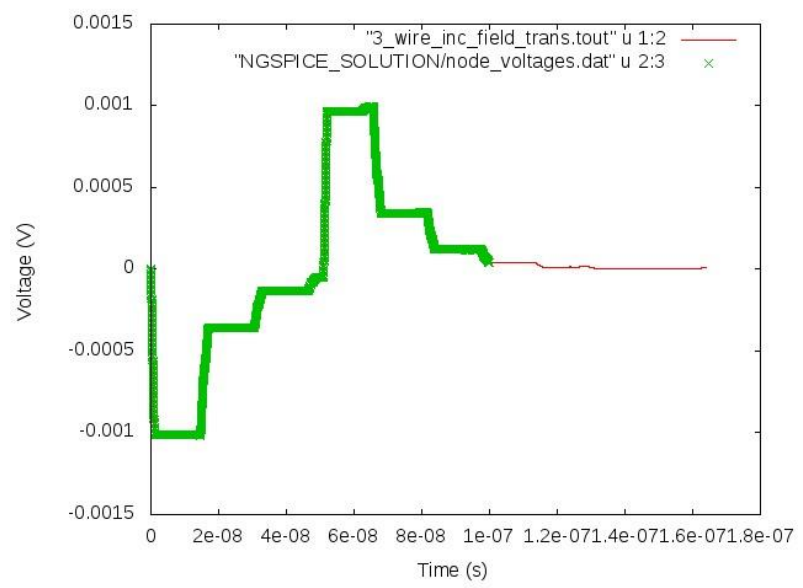

Figure 14. Coupling of a pulse plane wave onto a three wire transmission line.

Finally a test case configuration considering the crosstalk between two parallel coaxial cables was looked at. The cable specifications are listed in Tab.3 and the results are given in Fig. 15.

Two parallel coaxial cables
\begin{tabular}{|l|l|}
\hline Inner conductor radius & $0.455 \mathrm{~mm}$ \\
\hline Shield radius & $1.75 \mathrm{~mm}$ \\
\hline radius of outer dielectric & $2.5 \mathrm{~mm}$ \\
\hline relative permittivity of inner dielectric & 2.35 \\
\hline relative permittivity of outer dielectric & 3.0 \\
\hline coax cable separation & $1 \mathrm{~cm}$ \\
\hline transmission line length & $1.22 \mathrm{~m}$ \\
\hline Inner coax termination resistance, $\mathrm{R}$ & $50 \Omega$ \\
\hline Transfer resistance, $\mathrm{R}_{\mathrm{T}}$ & $0 \Omega$ \\
\hline Transfer inductance, $\mathrm{L}_{\mathrm{T}}$ & $2.5 \mathrm{nH} / \mathrm{m}$ \\
\hline
\end{tabular}

Table 3. Cable specification for two parallel coaxial cables.

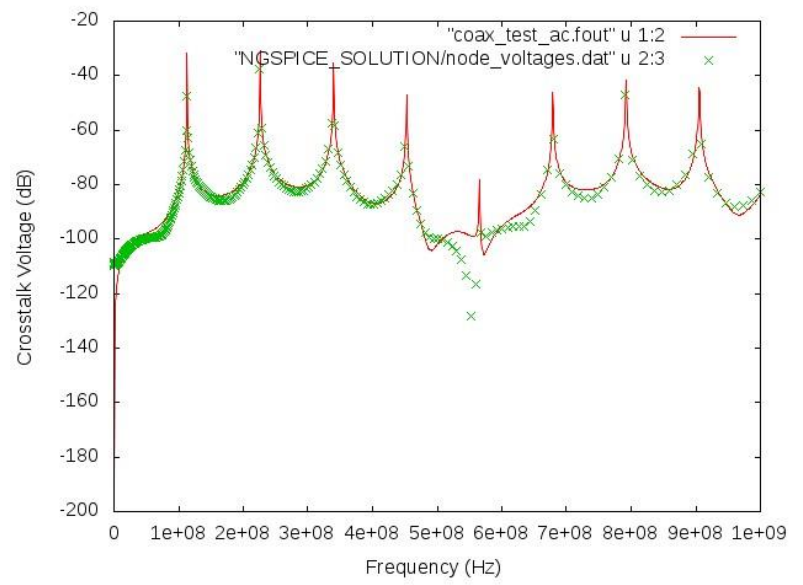

Figure 15. Crosstalk voltage as a function of frequency. 
The crosstalk voltage as a function of frequency shows distinct peaks where the crosstalk is enhanced by resonance in the circuit formed by the two shields, short circuited at either end. The agreement between the results is reasonable, but we note that this may be improved by implementation of a more rigorous model for coupling through two levels of shielding.

\section{CONCLUSION}

The requirements for modelling multi-conductor cable bundles for EMI, in particular for space applications, have been identified and suitable assumptions have been established that lead to viable Spice cable bundle model. During this work the incorporation of frequency dependent cable characteristics into the models has been found to be significant and one which most developers of Spice cable models have neglected in their models to date.

ACKNOWLEDGEMENT: This works is supported through the ESA project "Open Source Cable Models for EMI Simulations". ESA contract number: 4000112765/14/NL/HK.

\section{REFERENCES}

[1] C. R. Paul, "Analysis of Multiconductor Transmission Lines", Second edition John Wiley \& Sons, 2008, ISBN 978-0-470-13154-1.

[2] C. R. Paul, "A SPICE Model for Multiconductor Transmission Lines Excited by an Incident Electromagnetic Field," IEEE Trans EMC, Vol 36, No 4, 1994.

[3] H. Xie, J. Wang, R. Fan, Y. Liu, "SPICE Models to Analyze Radiated and Conducted Susceptibilities of Shielded Coaxial Cables," IEEE Trans EMC, Vol 52, No 1, 2010.

[4] C. R. Paul, "A SPICE Model for Multiconductor Transmission Lines Excited by an Incident Electromagnetic Field," IEEE Trans EMC, Vol 36, No 4, 1994. 